\title{
Periodic solutions for a kind of prescribed mean curvature Liénard equation with a singularity and a deviating argument
}

Shiping $\mathrm{Lu}^{1}$ and Fanchao Kong ${ }^{2 *}$

"Correspondence:
fanchaokong88@163.com
${ }^{2}$ Department of Mathematics,
Anhui Normal University, Wuhu,
241000, China
Full list of author information is
available at the end of the article

\begin{abstract}
In this paper, we study the existence of periodic solutions to the following prescribed mean curvature Liénard equation with a singularity and a deviating argument:

$$
\left(\frac{u^{\prime}(t)}{\sqrt{1+\left(u^{\prime}(t)\right)^{2}}}\right)^{\prime}+f(u(t)) u^{\prime}(t)+g(u(t-\sigma))=e(t),
$$

where $g$ has a strong singularity at $x=0$ and satisfies a small force condition at $x=\infty$. By applying Mawhin's continuation theorem, we prove that the given equation has at least one positive $T$-periodic solution. We will also give an example to illustrate the application of our main results.
\end{abstract}

Keywords: periodic solution; continuation theorem; prescribed mean curvature Liénard equation; deviating argument; singularity

\section{Introduction}

In recent years, there have been many papers about the existence of periodic solutions for the second order differential equations with a singularity, especially for the Liénard equations. And the existence of periodic solutions of the Liénard equations with a deviating argument has also been studied widely (see [1-12]). For example, in [2], Zhang studied the following Liénard equation with a singularity:

$$
x^{\prime \prime}(t)+f(x(t)) x^{\prime}(t)+g(t, x(t))=0,
$$

where $f: R \rightarrow R, g: R \times(0,+\infty) \rightarrow R$ is an $L^{2}$-Carathéodory function, $g(t, x)$ is a $T$-periodic function in the first argument and can be singular at $x=0$, i.e., $g(t, x)$ can be unbounded as $x \rightarrow 0^{+}$. Equation (1.1) is of repulsive type (resp. attractive type) if $g(t, x) \rightarrow-\infty$ (resp. $g(t, x) \rightarrow+\infty)$ for $x \rightarrow 0^{+}$.

Let Eq. (1.1) be of repulsive type and set

$$
\bar{g}(x)=\frac{1}{T} \int_{0}^{T} g(t, x) d t, \quad x>0 .
$$

Assume that

$$
\varphi(t)=\lim _{x \rightarrow+\infty} \sup \frac{g(t, x)}{x}
$$

(c) 2015 Kong and Lu; licensee Springer. This article is distributed under the terms of the Creative Commons Attribution 4.0 International License (http://creativecommons.org/licenses/by/4.0/), which permits unrestricted use, distribution, and reproduction in any medium, provided you give appropriate credit to the original author(s) and the source, provide a link to the Creative Commons license, and indicate if changes were made. 
exists uniformly for a.e. $t \in[0, T]$, i.e., for any $\varepsilon>0$, there is $g_{\varepsilon} \in L^{2}(0, T)$ such that

$$
g(t, x) \leq(\varphi(t)+\varepsilon) x+g_{\varepsilon}
$$

for all $x>0$ and a.e. $t \in[0, T]$. Assume that $\varphi \in C(R, R)$ and $\varphi(t+T)=\varphi(t), t \in R$.

Theorem 1.1 Assume that the following conditions are satisfied:

$\left(\mathrm{h}_{1}\right)$ (Balance condition) There exist constants $0<D_{1}<D_{2}$ such that if $x$ is a positive continuous T-periodic function satisfying

$$
\int_{0}^{T} g(t, x(t)) d t=0
$$

then

$$
D_{1} \leq x(\tau) \leq D_{2} \quad \text { for some } \tau \in[0, T]
$$

$\left(\mathrm{h}_{2}\right)$ (Degree condition) $\bar{g}(x)<0$ for all $x \in\left(0, D_{1}\right)$, and $\bar{g}(x)>0$ for all $x>D_{2}$.

$\left(\mathrm{h}_{3}\right)$ (Decomposition condition) $g(t, x)=g_{0}(x)+g_{1}(t, x)$, where $g_{0} \in C((0,+\infty), R)$ and $g_{1}$ : $[0, T] \times[0,+\infty) \rightarrow R$ is an $L^{2}$-Carathéodory function, i.e., $g_{1}$ is measurable with respect to the first variable, continuous with respect to the second one, and for any $b>0$ there is $h_{b} \in L^{2}((0, T) ;[0,+\infty))$ such that $\left|g_{1}(t, x)\right| \leq h_{b}(t)$ for a.e. $t \in[0, T]$ and all $x \in[0, b]$.

$\left(\mathrm{h}_{4}\right)$ (Strong force condition at $\left.x=0\right) \int_{0}^{1} g_{0}(x) d x=-\infty$.

$\left(\mathrm{h}_{5}\right)$ (Small force condition at $x=\infty$ )

$$
\left\|\varphi^{+}\right\|_{1}<\frac{\sqrt{3}}{T} \quad\left(\varphi^{+}=\max \left\{\varphi^{+}, 0\right\}\right) .
$$

Then Eq. (1.1) has at least one positive T-periodic solution.

Moreover, we notice that in [10] Wang further studied the Liénard equation with a singularity and a deviating argument of the form

$$
x^{\prime \prime}(t)+f(x(t)) x^{\prime}(t)+g(t, x(t-\sigma))=0,
$$

where $0 \leq \sigma<T$ is a constant, $f: R \rightarrow R, g: R \times(0,+\infty) \rightarrow R$ is an $L^{2}$-Carathéodory function, $g(t, x)$ is a $T$-periodic function in the first argument and can be singular at $x=0$, i.e., $g(t, x)$ can be unbounded as $x \rightarrow 0^{+}$.

Theorem 1.2 Assume that the following conditions are satisfied:

$\left(\mathrm{h}_{1}\right)$ (Balance condition) There exist constants $0<D_{1}<D_{2}$ such that if $x$ is a positive continuous T-periodic function satisfying

$$
\int_{0}^{T} g(t, x(t)) d t=0
$$

then

$$
D_{1} \leq x(\tau) \leq D_{2} \text { for some } \tau \in[0, T] .
$$


$\left(\mathrm{h}_{2}\right)$ (Degree condition) $\bar{g}(x)<0$ for all $x \in\left(0, D_{1}\right)$, and $\bar{g}(x)>0$ for all $x>D_{2}$.

$\left(\mathrm{h}_{3}\right)$ (Decomposition condition) $g(t, x)=g_{0}(x)+g_{1}(t, x)$, where $g_{0} \in C((0,+\infty), R)$ and $g_{1}$ : $[0, T] \times[0,+\infty) \rightarrow R$ is an $L^{2}$-Carathéodory function, i.e., $g_{1}$ is measurable with respect to the first variable, continuous with respect to the second one, and for any $b>0$ there is $h_{b} \in L^{2}((0, T) ;[0,+\infty))$ such that $\left|g_{1}(t, x)\right| \leq h_{b}(t)$ for a.e. $t \in[0, T]$ and all $x \in[0, b]$.

$\left(\mathrm{h}_{4}\right)$ (Strong force condition at $\left.x=0\right) \int_{0}^{1} g_{0}(x) d x=-\infty$.

$\left(\mathrm{h}_{5}^{\prime}\right)$ (Small force condition at $x=\infty$ )

$$
\|\varphi\|_{\infty}<\left(\frac{\sqrt{\pi}}{T}\right)^{2}
$$

Then Eq. (1.2) has at least one positive T-periodic solution.

Nowadays, the prescribed mean curvature $\left(\frac{u^{\prime}(t)}{\sqrt{1+\left(u^{\prime}(t)\right)^{2}}}\right)^{\prime}$ of a function $u(t)$ frequently appears in different geometry and physics (see [13-16]). For example, in [13], Obersnel studied the existence, regularity and stability properties of periodic solutions of a capillarity equation in the presence of lower and upper solutions

$$
-\left(\frac{u^{\prime}}{\sqrt{1+u^{\prime 2}}}\right)=f(t, u)
$$

This equation, together with its $N$-dimensional counterpart

$$
-\operatorname{div}\left(\frac{\nabla u}{\sqrt{1+|\nabla u|^{2}}}\right)=f(x, u)
$$

plays an important role in various physical and geometrical questions. And the existence of the periodic solutions and homoclinic solutions for the prescribed curvature mean equation also attracts many authors' attention. In [17], Feng discussed the periodic solution for the prescribed mean curvature Liénard equation of the form

$$
\left(\frac{u^{\prime}(t)}{\sqrt{1+\left(u^{\prime}(t)\right)^{2}}}\right)^{\prime}+f(u(t)) u^{\prime}(t)+g(t, u(t-\tau(t)))=e(t),
$$

estimated a priori bounds by eliminating the nonlinear term $\left(\frac{u^{\prime}(t)}{\sqrt{1+u^{\prime}(t)^{2}}}\right)^{\prime}$, and established sufficient conditions on the existence of periodic solutions by using Mawhin's continuation theorem. Moreover, Liang and $\mathrm{Lu}$ [18] studied the homoclinic solution for the prescribed mean curvature Duffing-type equation of the form

$$
\left(\frac{u^{\prime}(t)}{\sqrt{1+\left(u^{\prime}(t)\right)^{2}}}\right)^{\prime}+c u^{\prime}(t)+f(u(t))=p(t)
$$

where $f \in C^{1}(R, R), p \in C(R, R), c>0$ is a given constant.

However, to the best of our knowledge, the studying of periodic solutions for the prescribed mean curvature equation with a singularity is relatively infrequent, and the method of finding a priori bounds is different from the other prescribed mean curvature equations which have no singularities. So, it is worthwhile and interesting to explore this topic. 
In this paper, we consider the following prescribed mean curvature Liénard equation with a singularity and a deviating argument:

$$
\left(\frac{u^{\prime}(t)}{\sqrt{1+\left(u^{\prime}(t)\right)^{2}}}\right)^{\prime}+f(u(t)) u^{\prime}(t)+g(u(t-\sigma))=e(t)
$$

where $0 \leq \sigma<T, g:(0,+\infty) \rightarrow R$ is a continuous function and can be singular at $u=0$, i.e., $g(u)$ can be unbounded as $u \rightarrow 0^{+} . e(t)$ is $T$-periodic with $\int_{0}^{T} e(t) d t=0$. By applying Mawhin's continuation theorem, we prove that Eq. (1.5) has at least one positive $T$-periodic solution. The interest is that the conditions imposed on $f, g$ and the approaches to estimate $a$ priori bounds of periodic solutions are not only different from (1.1) and (1.2) but also different from (1.3) and (1.4). At last, a numerical example demonstrates the validity of the method. To sum up, our results are essentially new.

The structure of the rest of this paper is as follows. In Section 2, we state some necessary definitions and lemmas. In Section 3, we prove the main result. Finally, we give an example of an application in Section 4.

\section{Preliminary}

In order to use Mawhin's continuation theorem, we first recall it.

Let $X$ and $Y$ be two Banach spaces, a linear operator $L: D(L) \subset X \rightarrow Y$ is said to be a Fredholm operator of index zero provided that

(a) $\operatorname{Im} L$ is a closed subset of $Y$,

(b) $\operatorname{dim} \operatorname{Ker} L=\operatorname{codim} \operatorname{Im} L<\infty$.

Let $X$ and $Y$ be two Banach spaces, $\Omega \subset X$ be an open and bounded set, and $L: D(L) \subset$ $X \rightarrow Y$ be a Fredholm operator of index zero. A continuous operator $N: \Omega \subset X \rightarrow Y$ is said to be $L$-compact in $\bar{\Omega}$ provided that

(c) $K_{p}(I-Q) N(\bar{\Omega})$ is a relative compact set of $X$,

(d) $Q N(\bar{\Omega})$ is a bounded set of $Y$,

where we define $X_{1}=\operatorname{Ker} L, Y_{2}=\operatorname{Im} L$, then we have the decompositions $X=X_{1} \oplus X_{2}$, $Y=Y_{1} \oplus Y_{2}$. Let $P: X \rightarrow X_{1}, Q: Y \rightarrow Y_{1}$ be continuous linear projectors (meaning $P^{2}=P$ and $\left.Q^{2}=Q\right)$, and $K_{p}=\left.L\right|_{\operatorname{Ker} P \cap D(L)} ^{-1}$.

Lemma 2.1 [19] Let $X$ and $Y$ be two real Banach spaces, $\Omega$ be an open and bounded set of $X$, and $L: D(L) \subset X \rightarrow Y$ be a Fredholm operator of index zero. The operator $N: \bar{\Omega} \subset X \rightarrow$ $Y$ is said to be L-compact in $\bar{\Omega}$. In addition, if the following conditions hold:

(1) $L x \neq \lambda N x, \forall(x, \lambda) \in \partial \Omega \times(0,1)$;

(2) $Q N x \neq 0, \forall x \in \operatorname{Ker} L \cap \partial \Omega$;

(3) $\operatorname{deg}\{J Q N, \Omega \cap \operatorname{Ker} L, 0\} \neq 0$, where $J: \operatorname{Im} Q \rightarrow \operatorname{Ker} L$ is a homeomorphism,

then $L x=N x$ has at least one solution in $D(L) \cap \bar{\Omega}$.

In order to use Lemma 2.1, let us consider the problem

$$
\left\{\begin{array}{l}
u^{\prime}(t)=\phi(v(t))=\frac{v(t)}{\sqrt{1-v^{2}(t)}} \\
v^{\prime}(t)=-f(u(t)) \phi(v(t))-g(u(t-\sigma))+e(t) .
\end{array}\right.
$$

Obviously, if $(u(t), v(t))^{\top}$ is a solution of (2.1), then $u(t)$ is a solution of (1.1). 
Let

$$
X=Y=\left\{x: x(t)=(u(t), v(t))^{\top} \in C^{1}\left(R, R^{2}\right), x(t)=x(t+T)\right\},
$$

where the normal $\|x\|=\max \left\{\|u\|_{0},\|v\|_{0}\right\}$, and $\|u\|_{0}=\max _{t \in[0, T]}|u|,\|v\|_{0}=\max _{t \in[0, T]}|v|$. It is obvious that $X$ and $Y$ are Banach spaces.

Now we define the operator

$$
L: D(L) \subset X \rightarrow Y, \quad L x=x^{\prime}=\left(u^{\prime}(t), v^{\prime}(t)\right)^{\top},
$$

where $D(L)=\left\{x \mid x=(u(t), v(t))^{\top} \in C^{1}\left(R, R^{2}\right), x(t)=x(t+T)\right\}$.

Let $X_{0}=\left\{x=(u(t), v(t))^{\top} \in C^{1}(R, R \times(-1,1)), x(t)=x(t+T)\right\}$. Define a nonlinear operator $N: \bar{\Omega} \subset\left(X \cap X_{0}\right) \subset X \rightarrow Y$ as follows:

$$
N x=\left(\frac{v(t)}{\sqrt{1-v^{2}(t)}},-f(u(t)) \frac{v(t)}{\sqrt{1-v^{2}(t)}}-g(u(t-\sigma))+e(t)\right)^{\top},
$$

where $\bar{\Omega} \subset X_{0} \subset X$ and $\Omega$ is an open and bounded set. Then problem (2.1) can be written as $L x=N x$ in $\bar{\Omega}$.

We know

$$
\operatorname{Ker} L=\left\{x \mid x \in X, x^{\prime}=\left(u^{\prime}(t), v^{\prime}(t)\right)^{\top}=(0,0)^{\top}\right\},
$$

then $\forall t \in R$ we have $u^{\prime}(t)=0, v^{\prime}(t)=0$. Obviously, $u \in R, v \in R$, thus $\operatorname{Ker} L=R^{2}$, and it is also easy to prove that $\operatorname{Im} L=\left\{y \in Y, \int_{0}^{T} y(s) d s=0\right\}$. Therefore, $L$ is a Fredholm operator of index zero.

Let

$$
\begin{aligned}
& P: X \rightarrow \operatorname{Ker} L, \quad P x=\frac{1}{T} \int_{0}^{T} x(s) d s, \\
& Q: Y \rightarrow \operatorname{Im} Q, \quad Q y=\frac{1}{T} \int_{0}^{T} y(s) d s .
\end{aligned}
$$

Let $K_{p}=\left.L\right|_{\operatorname{Ker} L \cap D(L)} ^{-1}$, then it is easy to see that

$$
\left(K_{p} y\right)(t)=\int_{0}^{T} G_{k}(t, s) y(s) d s
$$

where

$$
G_{k}(t)= \begin{cases}\frac{s-T}{T}, & 0 \leq t \leq s \\ \frac{s}{T}, & s \leq t \leq T\end{cases}
$$

For all $\bar{\Omega}$ such that $\bar{\Omega} \subset\left(X \cap X_{0}\right) \subset X$, we have $K_{p}(I-Q) N(\bar{\Omega})$ is a relative compact set of $X, Q N(\bar{\Omega})$ is a bounded set of $Y$, so the operator $N$ is $L$-compact in $\bar{\Omega}$.

For the sake of convenience, we list the following assumptions which will be used by us in studying the existence of periodic solutions to Eq. (1.5) in Section 3. 
$\left(\mathrm{H}_{1}\right)$ There exist positive constants $D_{1}$ and $D_{2}$ with $D_{1}<D_{2}$ such that

(1) for each positive continuous $T$-periodic function $x(t)$ satisfying $\int_{0}^{T} g(x(t)) d t=0$, there exists a positive point $\tau \in[0, T]$ such that $D_{1} \leq x(\tau) \leq D_{2}$

(2) $g(x)<0$ for all $x \in\left(0, D_{1}\right)$ and $g(x)>0$ for all $x>D_{2}$.

$\left(\mathrm{H}_{2}\right) g(x(t))=g_{1}(x(t))+g_{0}(x(t))$, where $g_{1}:(0,+\infty) \rightarrow R$ is a continuous function and

(1) there exist positive constants $m_{0}$ and $m_{1}$ such that $g(x) \leq m_{0} x+m_{1}$ for all $x$ in $(0,+\infty)$

(2) $\int_{0}^{1} g_{0}(x) d x=-\infty$.

$\left(\mathrm{H}_{3}\right)$ There exist positive constants $\gamma, c_{0}, c_{1}$ such that $\gamma<f(x) \leq c_{0}|x|+c_{1}$ for all $x$ in $(0,+\infty)$.

Throughout this paper, define $A:=\left(\int_{0}^{T}|e(t)|^{2} d t\right)^{\frac{1}{2}}+\sup _{t \in[0, T]}|e(t)|<+\infty$.

\section{Existence of periodic solutions}

Theorem 3.1 Suppose that conditions $\left(\mathrm{H}_{1}\right)-\left(\mathrm{H}_{3}\right)$ hold and $\sigma=k T$, where $k$ is an integer. Also,

$$
\frac{A^{2} c_{0} T}{\gamma^{2}}+\frac{A \sqrt{T}\left(c_{0} D_{2}+c_{1}+2 m_{0} T\right)}{\gamma}+T\left(2 m_{0} D_{2}+2 m_{1}+A\right)<1 .
$$

Then, there exist positive constants $A_{1}, A_{2}$ and $\rho$, which are independent of $\lambda$ such that

$$
A_{1}<u(t)<A_{2}, \quad\|v\|_{0}<\rho<1,
$$

where $u(t)$ is any solution to the equation $L z=\lambda N z, \lambda \in(0,1)$.

Proof Let $\Omega_{1}=\{z \in \bar{\Omega}, L z=\lambda N z, \lambda \in(0,1)\}$. If $z \in \Omega_{1}$, we have

$$
\left\{\begin{array}{l}
u^{\prime}(t)=\lambda \phi(v(t))=\lambda \frac{v(t)}{\sqrt{1-v^{2}}(t)} \\
v^{\prime}(t)=-\lambda f(u(t)) \phi(v(t))-\lambda g(u(t-\sigma))+\lambda e(t)
\end{array}\right.
$$

Integrating the second equation of (3.1) from 0 to $T$, we have

$$
\int_{0}^{T} g(u(t-\sigma)) d t=0
$$

Combining with $\left(\mathrm{H}_{1}\right)(1)$, we can see that there exist positive constants $D_{1}, D_{2}$ and $\tau \in[0, T]$ such that

$$
D_{1} \leq u(\tau) \leq D_{2}
$$

Therefore, we have

$$
\begin{aligned}
\|u\|_{0} & =\max _{t \in[0, T]}|u(t)| \\
& \leq \max _{t \in[0, T]}\left|u(\tau)+\int_{\tau}^{t} u^{\prime}(s) d s\right|
\end{aligned}
$$




$$
\begin{aligned}
& \leq D_{2}+\int_{0}^{T}\left|u^{\prime}(s)\right| d s \\
& \leq D_{2}+\sqrt{T}\left\|u^{\prime}\right\|_{2} .
\end{aligned}
$$

Multiplying the second equation of (3.1) by $u^{\prime}(t)$ and integrating on the interval $[0, T]$, we have

$$
\begin{aligned}
0 & =\int_{0}^{T} v^{\prime}(t) u^{\prime}(t) d t \\
& =-\lambda \int_{0}^{T} f(u(t))\left(u^{\prime}(t)\right)^{2} d t-\lambda \int_{0}^{T} g(u(t-\sigma)) u^{\prime}(t) d t+\lambda \int_{0}^{T} e(t) u^{\prime}(t) d t \\
& =-\lambda \int_{0}^{T} f(u(t))\left(u^{\prime}(t)\right)^{2} d t-\lambda \int_{0}^{T} g(u(t-\sigma)) d u(t-\sigma)+\lambda \int_{0}^{T} e(t) u^{\prime}(t) d t \\
& =-\lambda \int_{0}^{T} f(u(t))\left(u^{\prime}(t)\right)^{2} d t+\lambda \int_{0}^{T} e(t) u^{\prime}(t) d t .
\end{aligned}
$$

It follows from $\left(\mathrm{H}_{3}\right)$ that

$$
\gamma \int_{0}^{T}\left|u^{\prime}(t)\right|^{2} d t \leq \int_{0}^{T}|e(t)|\left|u^{\prime}(t)\right| d t
$$

From the inequality above, we get

$$
\left\|u^{\prime}\right\|_{2} \leq \frac{A}{\gamma}
$$

Substituting (3.6) into (3.4), we obtain

$$
\|u\|_{0} \leq D_{2}+\frac{A \sqrt{T}}{\gamma}:=M_{1}
$$

Furthermore, from the second equation of (3.1), we can get

$$
\begin{aligned}
\int_{0}^{T}\left|v^{\prime}(t)\right| d t \leq & \lambda \int_{0}^{T}|f(u(t))|\left|u^{\prime}(t)\right| d t \\
& +\lambda \int_{0}^{T}|g(u(t-\sigma))| d t+\lambda \int_{0}^{T}|e(t)| d t .
\end{aligned}
$$

It follows from $\left(\mathrm{H}_{3}\right)$ that

$$
\begin{aligned}
\int_{0}^{T}\left|v^{\prime}(t)\right| d t \leq & c_{0} \int_{0}^{T}|u(t)|\left|u^{\prime}(t)\right| d t+c_{1} \int_{0}^{T}\left|u^{\prime}(t)\right| d t \\
& +\int_{0}^{T}|g(u(t-\sigma))| d t+\int_{0}^{T}|e(t)| d t \\
\leq & c_{0}\|u\|_{0} \sqrt{T}\left\|u^{\prime}\right\|_{2}+c_{1} \sqrt{T}\left\|u^{\prime}\right\|_{2} \\
& +\int_{0}^{T}|g(u(t-\sigma))| d t+A T .
\end{aligned}
$$


Write

$$
I_{+}=\{t \in[0, T]: g(u(t-\sigma)) \geq 0\} ; \quad I_{-}=\{t \in[0, T]: g(u(t-\sigma)) \leq 0\} .
$$

Then we can get from (3.2) and $\left(\mathrm{H}_{2}\right)(1)$ that

$$
\begin{aligned}
\int_{0}^{T}|g(u(t-\sigma))| d t & =\int_{I_{+}} g(u(t-\sigma)) d t-\int_{I_{-}} g(u(t-\sigma)) d t \\
& =2 \int_{I_{+}} g(u(t-\sigma)) d t \\
& \leq 2 m_{0} \int_{0}^{T} u(t-\sigma) d t+2 \int_{0}^{T} m_{1} d t \\
& \leq 2 m_{0} T\|u\|_{0}+2 T m_{1} .
\end{aligned}
$$

Substituting (3.9) into (3.8) and combining with (3.6) and (3.7), we obtain

$$
\begin{aligned}
\int_{0}^{T}\left|v^{\prime}(t)\right| d t \leq & c_{0}\|u\|_{0} \sqrt{T}\left\|u^{\prime}\right\|_{2}+c_{1} \sqrt{T}\left\|u^{\prime}\right\|_{2} \\
& +2 m_{0} T\|u\|_{0}+2 T m_{1}+A T \\
\leq & \frac{A^{2} c_{0} T}{\gamma^{2}}+\frac{A \sqrt{T}\left(c_{0} D_{2}+c_{1}+2 m_{0} T\right)}{\gamma}+T\left(2 m_{0} D_{2}+2 m_{1}+A\right) .
\end{aligned}
$$

Integrating the first equation of (3.1) on the interval $[0, T]$, we have

$$
\int_{0}^{T} \frac{v(t)}{\sqrt{1-v^{2}}(t)} d t=0
$$

Then we can see that there exists $\eta \in[0, T]$ such that $v(\eta)=0$. It implies that

$$
|v(t)|=\left|\int_{\eta}^{t} v^{\prime}(s) d s+v(\eta)\right| \leq \int_{0}^{T}\left|v^{\prime}(s)\right| d s .
$$

Combining with (3.10) gives

$$
\begin{aligned}
|v(t)| & \leq \int_{0}^{T}\left|v^{\prime}(s)\right| d s \\
& \leq \frac{A^{2} c_{0} T}{\gamma^{2}}+\frac{A \sqrt{T}\left(c_{0} D_{2}+c_{1}+2 m_{0} T\right)}{\gamma}+T\left(2 m_{0} D_{2}+2 m_{1}+A\right) \\
& :=\rho .
\end{aligned}
$$

Since $\frac{A^{2} c_{0} T}{\gamma^{2}}+\frac{A \sqrt{T}\left(c_{0} D_{2}+c_{1}+2 m_{0} T\right)}{\gamma}+T\left(2 m_{0} D_{2}+2 m_{1}+A\right)<1$, then we have

$$
\|v\|_{0}=\max _{t \in[0, T]}|v(t)| \leq \rho<1 .
$$

Then from (3.1) we can also have

$$
\left\|u^{\prime}\right\|_{0} \leq \max _{t \in[0, T]} \frac{|v(t)|}{\sqrt{1-v^{2}}(t)} \leq \frac{\rho}{1-\rho^{2}}:=B
$$


On the other hand, from the second equation of (3.1) and by $\left(\mathrm{H}_{2}\right)$, we can see that

$$
v^{\prime}(t+\sigma)=-\lambda f(u(t+\sigma)) u^{\prime}(t+\sigma)-\lambda\left[g_{1}(u(t))+g_{0}(u(t))\right]+\lambda e(t+\sigma) .
$$

Multiplying both sides of Eq. (3.14) by $u^{\prime}(t)$, we have

$$
\begin{aligned}
v^{\prime}(t+\sigma) u^{\prime}(t)= & -\lambda f(u(t+\sigma)) u^{\prime}(t+\sigma) u^{\prime}(t)-\lambda\left[g_{1}(u(t))+g_{0}(u(t))\right] u^{\prime}(t) \\
& +\lambda e(t+\sigma) u^{\prime}(t) .
\end{aligned}
$$

Let $\tau \in[0, T]$ be as in (3.3). For any $t \in[\tau, T]$, integrating Eq. (3.15) on the interval $[\tau, T]$, we have

$$
\begin{aligned}
\lambda \int_{u(\tau)}^{u(t)} g_{0}(u) d u= & \lambda \int_{\tau}^{t} g_{0}(u(t)) u^{\prime}(t) d t \\
= & -\int_{\tau}^{t} v^{\prime}(t+\sigma) u^{\prime}(t) d t-\lambda \int_{\tau}^{t} f(u(t+\sigma)) u^{\prime}(t+\sigma) u^{\prime}(t) d t \\
& -\lambda \int_{\tau}^{t} g_{1}(u(t)) u^{\prime}(t) d t+\lambda \int_{\tau}^{t} e(t+\sigma) u^{\prime}(t) d t .
\end{aligned}
$$

Then from the inequality above, we get

$$
\begin{aligned}
\left|\int_{u(\tau)}^{u(t)} g_{0}(u) d u\right|= & \left|\int_{\tau}^{t} g_{0}(u(t)) u^{\prime}(t) d t\right| \\
\leq & \int_{0}^{T}\left|v^{\prime}(t+\sigma)\right|\left|u^{\prime}(t)\right| d t+\int_{0}^{T}|f(u(t+\sigma))|\left|u^{\prime}(t+\sigma)\right|\left|u^{\prime}(t)\right| d t \\
& +\int_{0}^{T}\left|g_{1}(u(t))\right|\left|u^{\prime}(t)\right| d t+\int_{0}^{T}|e(t+\sigma)|\left|u^{\prime}(t)\right| d t .
\end{aligned}
$$

Set $F_{M_{1}}=\max _{|u| \leq M_{1}}|f(u)|$ and $G_{M_{1}}=\max _{|u| \leq M_{1}}\left|g_{1}(u)\right|$, then we have

$$
\begin{aligned}
& \int_{0}^{T}|f(u(t+\sigma))|\left|u^{\prime}(t+\sigma)\right|\left|u^{\prime}(t)\right| d t \\
& \quad \leq F_{M_{1}}\left(\int_{0}^{T}\left|u^{\prime}(t+\sigma)\right|^{2} d t\right)^{\frac{1}{2}}\left(\int_{0}^{T}\left|u^{\prime}(t)\right|^{2} d t\right)^{\frac{1}{2}} \\
& \quad=F_{M_{1}}\left\|u^{\prime}\right\|_{2}^{2}
\end{aligned}
$$

and

$$
\int_{0}^{T}\left|g_{1}(u(t))\right|\left|u^{\prime}(t)\right| d t \leq G_{M_{1}}\left\|u^{\prime}\right\|_{0} T
$$

Substituting (3.17) and (3.18) into (3.16) and combining with (3.6) and (3.13), we obtain

$$
\begin{aligned}
\left|\int_{u(\tau)}^{u(t)} g_{0}(u) d u\right| \leq & \left\|u^{\prime}\right\|_{0} \int_{0}^{T}\left|v^{\prime}(t+\sigma)\right| d t+F_{M_{1}}\left\|u^{\prime}\right\|_{2}^{2} \\
& +G_{M_{1}}\left\|u^{\prime}\right\|_{0} T+A\left\|u^{\prime}\right\|_{2}
\end{aligned}
$$




$$
\begin{aligned}
& \leq \rho B+\frac{F_{M_{1}} A^{2}}{\gamma^{2}}+G_{M_{1}} B T+\frac{A^{2}}{\gamma} \\
& <+\infty .
\end{aligned}
$$

According to $\left(\mathrm{H}_{2}\right)(2)$, we can see that there exists a constant $M_{2}>0$ such that, for $t \in[\tau, T]$,

$$
u(t) \geq M_{2}
$$

For the case $t \in[0, \tau]$, we can handle it similarly.

Let us define

$$
0<A_{1}=\min \left\{D_{1}, M_{2}\right\}
$$

and

$$
A_{2}=\max \left\{D_{2}, M_{1}\right\}
$$

then by (3.3), (3.7) and (3.19) we can obtain

$$
A_{1}<u(t)<A_{2} \text {. }
$$

Therefore, from (3.12) and (3.20), we can see that the proof of Theorem 3.1 is now complete.

Theorem 3.2 Assume that all the conditions in Theorem 3.1 hold, then Eq. (1.5) has at least one positive T-periodic solution.

Proof Set

$$
\Omega=\left\{x=(u, v)^{\top} \in X: A_{1}<u(t)<A_{2},\|v\|_{0}<\rho_{1}<\frac{\rho+1}{2}\right\} .
$$

Then the condition (1) of Lemma 2.1 is satisfied.

Suppose that there exists $x \in \partial \Omega \cap \operatorname{Ker} L$ such that $Q N x=\frac{1}{T} \int_{0}^{T} N x(s) d s=(0,0)^{\top}$, i.e.,

$$
\left\{\begin{array}{l}
\frac{1}{T} \int_{0}^{T} \frac{v(t)}{\sqrt{1-v^{2}(t)}} d t=0, \\
\frac{1}{T} \int_{0}^{T}\left[-f(u(t)) \frac{v(t)}{\sqrt{1-v^{2}(t)}}-g(u(t-\sigma))+e(t)\right] d t=0 .
\end{array}\right.
$$

Since $\operatorname{Ker} L=R^{2}$, and $u \in R, v \in R$ are constant, combining with the first equation of (3.21), we obtain

$$
v=0<\rho .
$$

From the second equation of (3.21), we have

$$
\frac{1}{T} \int_{0}^{T} g(u(t-\sigma)) d t=0
$$

From $\left(\mathrm{H}_{1}\right)(1)$ we can see that

$$
A_{1}<D_{1} \leq u(t) \leq D_{2}<A_{2}
$$


It is in contradiction to $x \in \partial \Omega$. So $\forall x \in \operatorname{Ker} L \cap \partial \Omega$, we have $Q N x \neq 0$. Then the condition (2) of Lemma 2.1 is satisfied.

In the following, we prove that the condition (3) of Lemma 2.1 is also satisfied.

Let

$$
z=A x=A\left(\begin{array}{l}
u \\
v
\end{array}\right)=\left(\begin{array}{c}
u-\frac{A_{1}+A_{2}}{2} \\
v
\end{array}\right)
$$

then we have

$$
x=z+\left(\begin{array}{c}
\frac{A_{1}+A_{2}}{2} \\
0
\end{array}\right) .
$$

Define $J: \operatorname{Im} Q \rightarrow \operatorname{Ker} L$ is a linear isomorphism,

$$
J(u, v)=\left(\begin{array}{c}
v \\
-u
\end{array}\right)
$$

and define

$$
H(\mu, x)=\mu A x+(1-\mu) J Q N x, \quad \forall(x, \mu) \in \Omega \times[0,1] .
$$

Then

$$
H(\mu, x)=\left(\begin{array}{c}
\mu u-\frac{\mu\left(A_{1}+A_{2}\right)}{2} \\
\mu \nu
\end{array}\right)+\frac{1-\mu}{T}\left(\begin{array}{c}
\int_{0}^{T}\left[\frac{f(u) v}{\sqrt{1-v^{2}}}+g(u)\right] d t \\
\int_{0}^{T} \frac{v}{\sqrt{1-v^{2}}} d t
\end{array}\right) .
$$

Now we claim that $H(\mu, x)$ is a homotopic mapping. Assume, by way of contradiction, that there exist $\mu_{0} \in[0,1]$ and $x_{0}=\left(\begin{array}{c}u_{0} \\ v_{0}\end{array}\right) \in \partial \Omega$ such that $H\left(\mu_{0}, x_{0}\right)=0$.

Substituting $\mu_{0}$ and $x_{0}$ into (3.22), we have

$$
H\left(\mu_{0}, x_{0}\right)=\left(\begin{array}{c}
\mu_{0} u_{0}-\frac{\mu_{0}\left(A_{1}+A_{2}\right)}{2}+\left(1-\mu_{0}\right) f\left(u_{0}\right) \frac{v_{0}}{\sqrt{1-v_{0}^{2}}}+\left(1-\mu_{0}\right) g\left(u_{0}\right) \\
\mu_{0} \nu_{0}+\left(1-\mu_{0}\right) \frac{v_{0}}{\sqrt{1-v_{0}^{2}}}
\end{array}\right) .
$$

Since $H\left(\mu_{0}, x_{0}\right)=0$, then we can see that

$$
\mu_{0} v_{0}+\left(1-\mu_{0}\right) \frac{v_{0}}{\sqrt{1-v_{0}^{2}}}=0 .
$$

Combining with $\mu_{0} \in[0,1]$, we obtain $v_{0}=0$. Thus $u_{0}=A_{1}$ or $A_{2}$.

If $u_{0}=A_{1}$, it follows from $\left(\mathrm{H}_{1}\right)(2)$ that $g\left(u_{0}\right)<0$, then substituting $v_{0}=0$ into (3.23), we have

$$
\begin{aligned}
\mu_{0} & u_{0}-\frac{\mu_{0}\left(A_{1}+A_{2}\right)}{2}+\left(1-\mu_{0}\right) f\left(u_{0}\right) \frac{v_{0}}{\sqrt{1-v_{0}^{2}}}+\left(1-\mu_{0}\right) g\left(u_{0}\right) \\
& =\mu_{0} u_{0}-\frac{\mu_{0}\left(A_{1}+A_{2}\right)}{2}+\left(1-\mu_{0}\right) g\left(u_{0}\right) \\
& <\mu_{0}\left(u_{0}-\frac{A_{1}+A_{2}}{2}\right)<0 .
\end{aligned}
$$


If $u_{0}=A_{2}$, it follows from $\left(\mathrm{H}_{1}\right)(2)$ that $g\left(u_{0}\right)>0$, then substituting $v_{0}=0$ into (3.23), we have

$$
\begin{aligned}
& \mu_{0} u_{0}-\frac{\mu_{0}\left(A_{1}+A_{2}\right)}{2}+\left(1-\mu_{0}\right) f\left(u_{0}\right) \frac{v_{0}}{\sqrt{1-v_{0}^{2}}}+\left(1-\mu_{0}\right) g\left(u_{0}\right) \\
& =\mu_{0} u_{0}-\frac{\mu_{0}\left(A_{1}+A_{2}\right)}{2}+\left(1-\mu_{0}\right) g\left(u_{0}\right) \\
& >\mu_{0}\left(u_{0}-\frac{A_{1}+A_{2}}{2}\right)>0 .
\end{aligned}
$$

Combining with (3.24) and (3.25), we can see that $H\left(\mu_{0}, x_{0}\right) \neq 0$, which contradicts the assumption. Therefore $H(\mu, x)$ is a homotopic mapping and $x^{\top} H(\mu, x) \neq 0, \forall(x, \mu) \in(\partial \Omega \cap$ $\operatorname{Ker} L) \times[0,1]$. Then

$$
\begin{aligned}
\operatorname{deg}(J Q N, \Omega \cap \operatorname{Ker} L, 0) & =\operatorname{deg}(H(0, x), \Omega \cap \operatorname{Ker} L, 0) \\
& =\operatorname{deg}(H(1, x), \Omega \cap \operatorname{Ker} L, 0) \\
& =\operatorname{deg}(A x, \Omega \cap \operatorname{Ker} L, 0) \\
& =\sum_{x \in A^{-1}(0)} \operatorname{sgn}\left|A^{\prime}(x)\right| \\
& =1 \neq 0 .
\end{aligned}
$$

Thus, the condition (3) of Lemma 2.1 is also satisfied.

Therefore, by applying Lemma 2.1, we can conclude that Eq. (1.5) has at least one positive $T$-periodic solution.

\section{Example}

As an application, we consider the following example:

$$
\left(\frac{u^{\prime}(t)}{\sqrt{1+\left(u^{\prime}(t)\right)^{2}}}\right)^{\prime}+\left[\frac{u^{3}(t)}{3+u^{2}(t)}+9\right] u^{\prime}(t)+g(u(t))=\frac{1}{32} \sin 8 t .
$$

Corresponding to Theorem 3.1 and (1.5), we have

$$
\begin{aligned}
& f(u(t))=\frac{u^{3}(t)}{3+u^{2}(t)}+9, \quad g(u(t))=g_{1}(u(t))+g_{0}(u(t))=\frac{1}{32} u(t)-\frac{1}{u(t)}, \\
& e(t)=\frac{1}{32} \sin 8 t
\end{aligned}
$$

Then we can have and choose

$$
\begin{aligned}
& T=\frac{\pi}{4}, \quad \gamma=8, \quad c_{0}=\frac{1}{3}, \quad c_{1}=9, \\
& m_{0}=\frac{1}{32}, \quad m_{1}=\frac{1}{128}, \quad D_{1}=1, \quad D_{2}=9
\end{aligned}
$$

and

$$
A:=\left(\int_{0}^{T}|e(t)|^{2} d t\right)^{\frac{1}{2}}+\sup _{t \in[0, T]}|e(t)|<\frac{1}{16}<+\infty
$$


Then we can see that $\left(\mathrm{H}_{1}\right)(1)$ and $\left(\mathrm{H}_{1}\right)(2)$ hold. Meanwhile,

$$
\frac{A^{2} c_{0} T}{\gamma^{2}}+\frac{A \sqrt{T}\left(c_{0} D_{2}+c_{1}+2 m_{0} T\right)}{\gamma}+T\left(2 m_{0} D_{2}+2 m_{1}+A\right) \approx 0.587<1 .
$$

Hence, by applying Theorem 3.2, we can see that Eq. (4.1) has at least one positive $T$-periodic solution.

\section{Competing interests}

The authors declare that they have no competing interests.

\section{Authors' contributions}

All authors have equally contributed to obtaining new results in this article and also read and approved the final manuscript.

\section{Author details}

${ }^{1}$ College of Mathematics and Statistics, Nanjing University of Information Science and Technology, Nanjing, 210044, China. ${ }^{2}$ Department of Mathematics, Anhui Normal University, Wuhu, 241000, China.

\section{Acknowledgements}

The work was supported by the National Natural Science Foundation of China (Grant No. 11271197) and the Ministry of Education Key Project of Natural Science (207047).

\section{Received: 25 February 2015 Accepted: 14 April 2015 Published online: 09 May 2015}

\section{References}

1. Fonda, A, Manásevich, R, Zanolin, F: Subharmonic solutions for some second order differential equations with singularities. SIAM J. Math. Anal. 24, 1294-1311 (1993)

2. Zhang, M: Periodic solutions of Liénard equations with singular forces of repulsive type. J. Math. Anal. Appl. 203 254-269 (1996)

3. Torres, PJ: Existence of one-signed periodic solutions of some second order differential equations via a Krasnoselskii fixed point theorem. J. Differ. Equ. 190, 643-662 (2003)

4. Jiang, D, Chu, J, Zhang, M: Multiplicity of positive periodic solutions to superlinear repulsive singular equations. J. Differ. Equ. 211, 282-302 (2005)

5. Lazer, AC, Solimini, S: On periodic solutions of nonlinear differential equations with singularities. Proc. Am. Math. Soc. 88, 109-114 (1987)

6. Li, X, Zhang, Z: Periodic solutions for second order differential equations with a singular nonlinearity. Nonlinear Anal. TMA 69, 3866-3876 (2008)

7. Hakl, R, Torres, PJ, Zamora, M: Periodic solutions to singular second order differential equations: the repulsive case. Nonlinear Anal. TMA 74, 7078-7093 (2011)

8. Wang, Z: Periodic solutions of the second order differential equations with singularities. Nonlinear Anal. TMA 58, 319-331 (2004)

9. Wang, Z, Ma, T: Existence and multiplicity of periodic solutions of semilinear resonant Duffing equations with singularities. Nonlinearity 25, 279-307 (2012)

10. Wang, Z: Periodic solutions of Liénard equation with a singularity and a deviating argument. Nonlinear Anal., Real World Appl. 16, 227-234 (2014)

11. Liu, B, Huang, L: Existence and uniqueness of periodic solutions for a kind of Liénard equation with a deviating argument. Appl. Math. Lett. 21, 56-62 (2008)

12. Lu, S, Ge, W: Periodic solutions for a kind of Liénard equation with a deviating argument. J. Math. Anal. Appl. 289, 231-243 (2004)

13. Obersnel, F: Existence, regularity and stability properties of periodic solutions of a capillarity equation in the presence of lower and upper solutions. Nonlinear Anal., Real World Appl. 13, 2830-2852 (2012)

14. Bonheure, D, Habets, P, Obersnel, F, Omari, P: Classical and non-classical solutions of a prescribed curvature equation. J. Differ. Equ. 243, 208-237 (2007)

15. Pan, $\mathrm{H}$ : One-dimensional prescribed mean curvature equation with exponential nonlinearity. Nonlinear Anal. 70 999-1010 (2009)

16. Benevieria, P, do Ó, JM, de Medeiros, ES: Periodic solutions for nonlinear systems with mean curvature-like operators. Nonlinear Anal. 65, 1462-1475 (2006)

17. Feng, M: Periodic solutions for prescribed mean curvature Liénard equation with a deviating argument. Nonlinear Anal., Real World Appl. 13, 1216-1223 (2012)

18. Liang, Z, Lu, S: Homoclinic solutions for a kind of prescribed mean curvature Duffing-type equation. Adv. Differ. Equ. 2013, $279(2013)$

19. Gaines, RE, Mawhin, JL: Coincidence Degree and Nonlinear Differential Equations. Springer, Berlin (1977) 